\title{
Stockage thermique de l'énergie par chaleur latente de fusion d'un sel minéral : étude d'un échangeur dynamique à contact direct avec cristallisation du sel durant l'écoulement
}

\author{
J. Pantaloni, O. Favre, R. Bailleux, G. Finiels et J. Marchisio \\ Laboratoire de Dynamique et Thermophysique des fluides $\left({ }^{*}\right)$, \\ Université de Provence, Centre de St-Jérôme, \\ rue Henri-Poincarré, 13397 Marseille Cedex 4, France
}

(Reçu le 12 juin 1978, accepté le 17 juillet 1978)

\begin{abstract}
Résumé. - Nous nous sommes proposés, dans le présent travail, d'étudier la possibilité de mettre en œuvre un échangeur à contact direct par injection d'un sel fondu (mélange eutectique de nitrate et de nitrite) dans une huile de synthèse (Gilotherm-A.L.D.) en écoulement turbulent à la température initiale inférieure au point de fusion du sel $T_{\mathrm{F}}=142^{\circ} \mathrm{C}$. Une première série d'expériences consistant à refroidir sous agitation contrôlée un mélange d'huile et de sel fondu démontre la faisabilité du système. Une deuxième série d'expériences, relative à l'injection du sel fondu dans l'huile, montre que la granulométrie obtenue est fonction de la vitesse d'injection et de la différence de température entre le fluide caloporteur et le sel et que le rendement de l'échange est de l'ordre de $85 \%$. Nous montrons ainsi qu'un échangeur à contact direct permet une récupération relativement aisée de la chaleur latente de fusion avec utilisation du matériau de stockage que durant la phase d'échange.
\end{abstract}

\begin{abstract}
We present here some experiments on the possibility to conceive a heat exchange with recuperation of latent heat of molten salt by injection of the molten salt (eutectic mixture of nitrate and nitrite) in a synthetic oil (Gilotherm-A.L.D.) in turbulent motion at a initial temperature inferior to melting point of salt $T_{\mathrm{F}}=142{ }^{\circ} \mathrm{C}$. A first experimental serie which consists to freeze a mixture of oil and molten salt with a controlled agitation gives a clear indication on the possibility to conceive this system. A second experimental serie which consists to an injection of the molten salt in oil shows that the size of solid particles obtained is a function of the injection velocity and of the temperature difference between oil and salt; these experiments show also that the exchange efficiency is about $85 \%$. In conclusion, this system allows an easy and optimal recuperation of latent heat of molten salt used for thermal energy storage.
\end{abstract}

1. Introduction. - Le stockage thermique de l'énergie constitue actuellement un des principaux thèmes de recherche dans le domaine de l'énergétique; il s'agit sans doute d'un des moyens les mieux adaptés à la modulation d'une source énergétique périodique permettant de disposer en sortie d'une puissance thermique constante.

Outre le stockage par chaleur sensible dont certaines réalisations existent déjà au niveau semiindustriel [1, 2], le stockage peut également utiliser la chaleur latente de changement d'état, particulièrement la chaleur latente de fusion qui permet la récupération à un niveau de température constant. C'est ainsi que dans le cas d'un mélange eutectique de nitrate et de nitrite $\left[\mathrm{NaNo}_{3}-\mathrm{NaNo}_{2}-\mathrm{KNo}_{3}\right]$ faisant l'objet du présent travail, l'énergie stockée sous forme de chaleur latente correspond à un stockage par chaleur sensible sur un intervalle de température de $50^{\circ} \mathrm{C}$.

(*) Laboratoire associé au C.N.R.S. no 72.
Toutefois, en l'état actuel de la technologie des échangeurs, la récupération de la chaleur latente présente de nombreuses difficultés et de nombreux travaux se développent dans ce domaine [3-14]. Ces études ont principalement trait au déstockage sous forme statique avec transfert thermique du stockage vers le fluide caloporteur à travers une croûte solide généralement mauvaise conductrice thermique. Le matériau de stockage est placé soit à l'intérieur de capsules [14] autour desquelles circule le fluide caloporteur, soit dans une enceinte parcourue de tubes dans lesquels circule le fluide [8-10-13]. Bien qu'une étude méthodologique [10] montre que la deuxième solution permette une meilleure récupération de l'énergie stockée, ces solutions sont toujours limitées par l'importance de la surface d'échange qu'il faut mettre en œuvre pour obtenir des rendements appréciables [15], ceci malgré les phénomènes de convection naturelle mis en jeu au niveau de l'interface solideliquide du matériau de stockage [11-12].

Afin de s'affranchir de la surface d'échange qui 
pénalise ce mode de stockage et peut en limiter le développement [15] pour les installations de fortes puissances, il semble que lorsque le domaine de température le permet et si les matériaux existent, le principe de l'échangeur dynamique à contact direct entre le matériau de stockage et le fluide caloporteur doit être retenu préférentiellement aux échangeurs statiques. Le principe est simple ; il consiste à pulvériser le matériau de stockage à l'état liquide directement dans le fluide caloporteur (liquide ou gaz) circulant soit à co-courant soit à contre-courant.

Outre le fait de posséder une surface d'échange importante par rapport au volume du matériau, ce type d'échangeur présente également l'avantage de n'utiliser le matériau que durant la phase d'échange. En effet, lorsque la particule a cédé son énergie de changement d'état au fluide caloporteur, elle est éliminée de l'enceinte de l'échangeur et n'encombre pas celui-ci inutilement comme dans l'échangeur statique.

Toutefois, la mise en œuvre d'un tel système nécessite l'existence de couples compatibles matériau de stockage-fluide caloporteur; en particulier, les deux composants doivent être chimiquement inertes et ne pas présenter de phénomènes de miscibilité tant à l'état solide qu'à l'état liquide. Diverses solutions sont possibles; c'est ainsi que dans le cadre des systèmes métaux-sels, nous avons étudié dans un précédent travail [16] l'injection de chlorure de sodium liquide (point de fusion $801{ }^{\circ} \mathrm{C}$ ) dans du sodium liquide. D'autres auteurs ont étudié [17] l'injection d'eau dans un courant de kérozène dont la température initiale est inférieure à $0^{\circ} \mathrm{C}$; il existe également la possibilité d'injection de chlorure de sodium liquide dans un courant gazeux [18].

Nous nous proposons dans le présent travail d'étudier un échangeur de ce type au niveau des divers processus mis en jeu : faisabilité, mécanisme de l'injection, rendement de l'échange. En ce qui concerne le matériau de stockage, nous avons choisi le mélange eutectique : $\mathrm{NaNo}_{2}-\mathrm{NaNo}_{3}-\mathrm{KNo}_{3}(40 \%, 7 \%, 53 \%)$ dont le point de fusion $\left(142^{\circ} \mathrm{C}\right)$ permet de réaliser des expériences de visualisation et correspond à un domaine de température susceptible d'application. Le fluide caloporteur est une huile organique de synthèse principalement constituée de Terphenyls (Gilotherm, A.L.D., Rhône-Poulenc).

2. Etude préalable statique et dynamique du système sel-huile. - Dans le cas des couples métaux-sel, on dispose généralement des diagrammes de phases même si ceux-ci ne sont pas toujours connus avec précision. C'est ainsi que pour le couple $\mathrm{NaCl}-\mathrm{Na}$, il est aisé de connaître la composition de la phase solide qui se forme à $796^{\circ} \mathrm{C}$ par refroidissement du mélange liquide binaire; le composé solide est principalement constitué de $\mathrm{NaCl}$ alors que le liquide est principalement du sodium. Dans ces conditions, ce couple répond relativement bien aux critères de l'inertie chimique et de l'absence de miscibilité. Nous avons alors montré expérimentalement que ce couple était acceptable et permettait une récupération de la chaleur latente de fusion du $\mathrm{NaCl}$ lors de la cristallisation en écoulement dans le sodium [16]. En ce qui concerne les mélanges sel minéral-huile organique, nous ne disposons pratiquement d'aucune donnée relative au comportement chimique de ce type de système, excepté dans le cas du couple soude-gilotherm où des études par analyse thermique différentielle [19] ne semblent pas favorables à l'utilisation de ce système.

Une étude préalable du système $\left[\mathrm{NaNo}_{3}-\mathrm{NaNo}_{2}-\right.$ $\mathrm{KNo}_{3}$, huile (Gilotherm, A.L.D.) consistant à chauffer à la température de $150^{\circ} \mathrm{C}$ des mélanges d'huile et d'eutectique ainsi que de l'huile seule a montré que la vitesse de vieillissement de l'huile était plus grande en présence de sel que lorsque ce composé était absent [20]; d'autres travaux devront se développer dans ce domaine afin de déterminer la durée de vie de ce système.

Pour notre part, nous avons conduit une série d'expériences préalables relatives à la possibilité de récupérer l'énergie de chaleur latente de fusion par contact direct et à la séparation des deux phases solide et liquide après l'échange [21]. La figure 1 donne le principe du montage expérimental; on

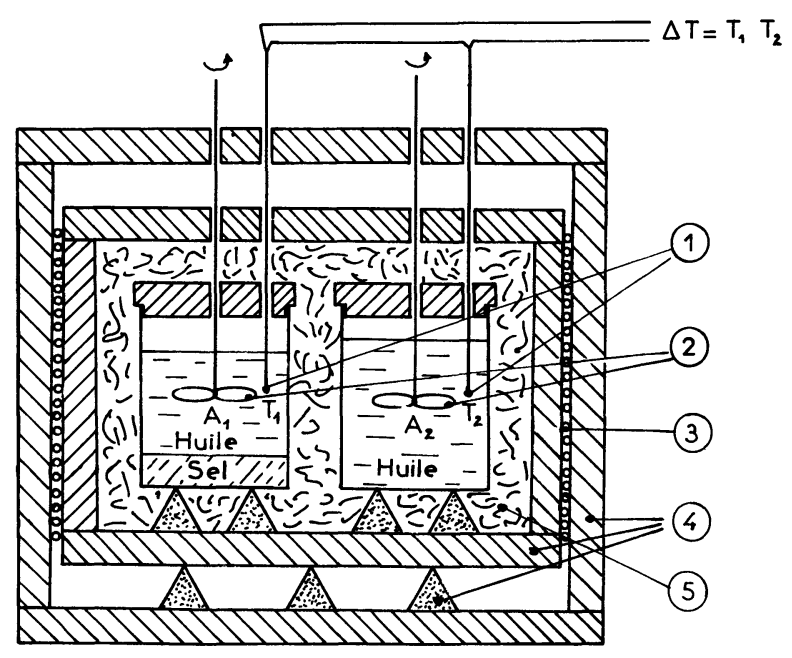

Fig. 1. - Deux enceintes absolument identiques sont placées dans un four régulé programmable. Dans l'enceinte 1 se trouve le mélange huile-sel fondu alors que dans l'enceinte 2 on ne place que de l'huile. L'étude du refroidissement de l'ensemble en fonction de la position de l'agitateur permet de simuler un échangeur statique avec convection libre, un échangeur statique avec convection forcée à l'interface et un échangeur dynamique à contact direct : 1) Thermocouples ; 2) Agitateurs ; 3) Enroulement chauffant ; 4) Enceinte en matériaux réfractaires.

[ Two similar containers are placed in an oven whose temperature is programmed. In the first container, there is a mixture oil-molten salt ; in the second there is only oil. The study of the temperature decrease as a function of the stirrer position allows a simulation of a static exchange with natural convection, a static exchange with forced convection and a dynamic exchange with direct contact : 1) Thermocouples ; 2) Stirrer ; 3) Warming ; 4) Refractory materials.] 
dispose d'un four régulé, programmable en température et de deux enceintes parfaitement identiques ; dans chaque enceinte, nous disposons d'un agitateur et d'un ensemble de thermocouples préalablement étalonnés. Dans une première série d'expériences, nous étalonnons l'ensemble en enregistrant simultanément la température $T_{1}$ dans l'enceinte 1 et la différence de température $\Delta T_{1}=T_{1}-T_{2}$ lorsqu'il y a la même quantité d'huile dans les deux enceintes; nous constatons qu'au cours d'un refroidissement programmé de $160^{\circ} \mathrm{C}$ à $120^{\circ} \mathrm{C}$, la différence $\Delta T_{1}$ reste pratiquement nulle attestant le comportement identique des deux enceintes. Dans une deuxième série d'expériences, nous plaçons dans la première enceinte un volume $V m$ d'huile et de sel à la concentration volumique en sel de $25 \%$ et dans la deuxième enceinte un volume $V$ d'huile pure tel que les capacités calorifiques initiales à $160{ }^{\circ} \mathrm{C}$ des deux enceintes soient sensiblement égales; cette condition impose le volume $V$ :

$V=\frac{V m}{4}\left(3+\frac{\rho_{\mathrm{s}} C_{\mathrm{s}}}{\rho_{\mathrm{h}} C_{\mathrm{h}}}\right)$

$\rho_{\mathrm{S}}\left(=1,96 \mathrm{~g} / \mathrm{cm}^{3}\right)$ et $\rho_{\mathrm{h}}\left(=0,78 \mathrm{~g} / \mathrm{cm}^{3}\right)$ étant les masses volumiques respectives du sel et de l'huile à $T=160^{\circ} \mathrm{C}, C_{\mathrm{S}}(=0,372 \mathrm{cal} / \mathrm{g} \mathrm{K})$ et $C_{\mathrm{h}}(=0,573 \mathrm{cal} /$ $\mathrm{g} \mathrm{K}$ ) les capacités calorifiques.

Nous étudions la cinétique de refroidissement de l'ensemble dans le domaine de température $\left(160^{\circ} \mathrm{C}\right.$ $120^{\circ} \mathrm{C}$ ) englobant la température de changement de phase du sel $\left(T_{\mathrm{F}}=142^{\circ} \mathrm{C}\right)$ en fonction de la position des agitateurs. Nous avons successivement considéré les trois situations suivantes :

1. Agitateur immobile en position haute. - Configuration simulant un échangeur statique à surface d'échange horizontale ne mettant en jeu que les processus d'échange par conduction et convection naturelle.

2. Agitateur en rotation en position haute. - On simule alors un échangeur statique mettant en jeu les processus d'échange par conduction et convection forcée à l'interface.

3. Agitateur en rotation en position basse. - On simule alors un échangeur dynamique.

2.1 Configuration 1 : Agitateur immobile. Nous enregistrons la température $T_{1}$ et la différence de température $\Delta T_{1}$ en fonction du temps. On ne constate (Fig. 2) aucun changement de pente caractéristique de la cristallisation du sel à $T_{\mathrm{F}} \simeq 142^{\circ} \mathrm{C}$. En effet, le thermocouple $T_{1}$ est situé à une distance de $2 \mathrm{~cm}$ de l'interface huile-sel, la diffusivité thermique de l'huile est de l'ordre de $10^{-3} \mathrm{~cm}^{2} / \mathrm{s}$ et, dans ces conditions, le temps caractéristique $\tau$, associé à la diffusion de la chaleur, est de l'ordre de $4000 \mathrm{~s}$; il est alors prévisible que l'accroissement de température dû à la transition de phase ne se détecte pas expérimentalement.

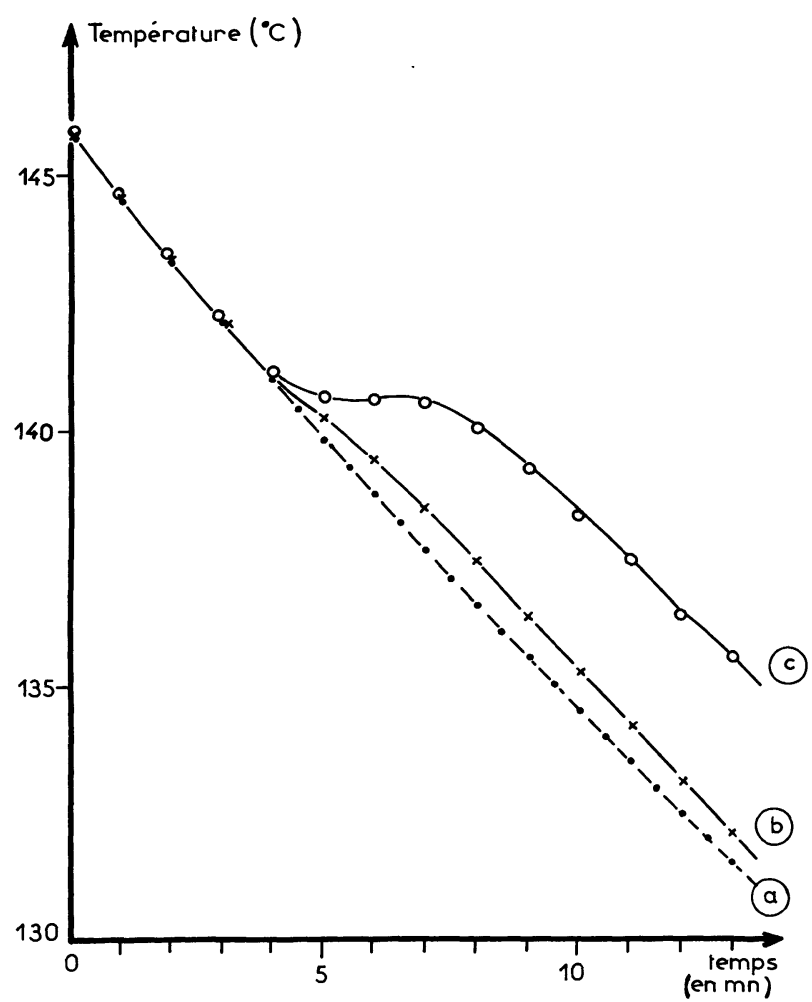

Fig. 2. - Courbes donnant la température de l'huile dans l'enceinte 1 en fonction de la position de l'agitateur : a) Agitateur immobile : échangeur statique sans convection forcée; b) Agitateur en position haute : échangeur statique avec convection forcée ; c) Agitateur à l'interface : échangeur dynamique à contact direct.

[Oil temperature dependance, in the first container, with the stirrer position. a) No motion of the stirrer : Simulation of a static heat exchanger with natural convection. b) Stirrer in high position : Simulation of a static heat exchanger with forced convection. c) Stirrer situated at the interface oil-molten salt : Simulation of a dynamic heat exchanger with direct contact.]

Dans cette configuration, les seuls mouvements animant les deux fluides sont dus à la convection naturelle et nous pouvons considérer que nous avons ainsi simulé un échangeur statique sans convection forcée. On remarque que la puissance délivrée par cet ensemble est pratiquement nulle.

2.2 Configuration 2 : Agitateur en rotation en position haute. - Nous avons placé l'agitateur dans une position déterminée au préalable de telle façon que les effets dus au vortex soient amortis au niveau de l'interface; nous avons ainsi vérifié qu'il n'y a pas déformation de l'interface qui reste plan durant toute la phase de refroidissement. Des résidus carbonés provenant de l'huile se déposent sur l'interface; ils nous permettent de visualiser les mouvements superficiels de l'interface. Nous avons constaté que cette configuration donne des vitesses pratiquement nulles.

Nous constatons sur la courbe de refroidissement (Fig. 2) un changement caractéristique au voisinage de $T \simeq 142{ }^{\circ} \mathrm{C}$. Du fait du brassage de l'huile, la chaleur latente dégagée par le sel perturbe sensiblement la cinétique du refroidissement. 
Nous adoptons alors comme référence le refroidissement en l'absence de rotation (configuration 1); nous déterminons l'écart de température

$$
\Delta T(t)=T_{1}(t)-T_{\text {ref }}(t)
$$

(Fig. 3) ainsi que la pente $\mathrm{d} \Delta T / \mathrm{d} t$ qui est proportionnelle à la puissance instantanée récupérée par l'huile (Fig. 4).

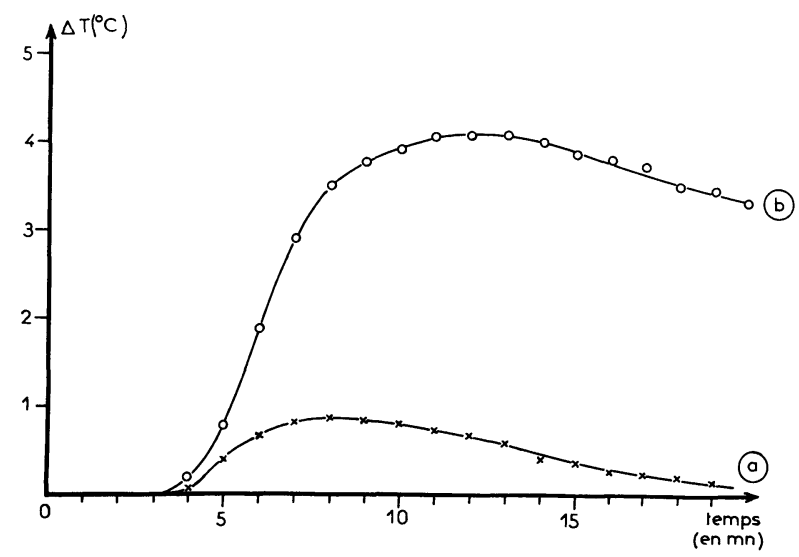

Fig. 3. - Accroissement de température de l'huile par rapport à la référence [courbe (a) de la figure 2] en fonction du temps; (a) Agitateur en position haute; (b) Agitateur au niveau de l'interface. On remarque que l'accroissement $\Delta T$ est maximum dans le cas de l'échange dynamique à contact direct.

[Oil temperature increase, in the first container, in reference to temperature obtained with motionless stirrer (Curve (a), Figure 2). (a) Stirrer in high position; (b) Stirrer at the interface. We see that the increase $\Delta T$ is maximum when there is direct contact.]

Cette configuration, du fait de la position de l'agitateur, simule un échangeur statique dans lequel le fluide caloporteur (huile) est animé d'un mouvement de rotation; le transfert thermique du sel vers l'huile est alors dû à la conduction et à la convection forcée. Nous constatons une récupération effective d'énergie durant un temps $t$ de l'ordre de 6 minutes avec un accroissement de température égal au maximum à $0,9{ }^{\circ} \mathrm{C}$.

2.3 Configuration $3:$ Agitateur en rotation en position basse. - L'agitateur est placé dans une position déterminée au préalable de telle façon qu'il y ait perturbation de l'interface huile-sel. Celui-ci se déforme légèrement pour devenir parabolique mais on constate principalement l'apparition d'ondulations dues à la perturbation verticale créé par l'agitateur. Cette situation, pour $T>T_{\mathrm{F}}\left(=142^{\circ} \mathrm{C}\right)$, n'engendre pas l'apparition sensible d'une zone émulsionnelle avec gouttelettes de sel liquide dans l'huile. Par contre, au voisinage du point de fusion du sel, on remarque l'apparition de cristallite en concentration croissant très rapidement en fonction du temps. Du point de vue de l'analyse physique de ce phénomène, il semblerait que nous soyons en présence d'une instabilité inter-

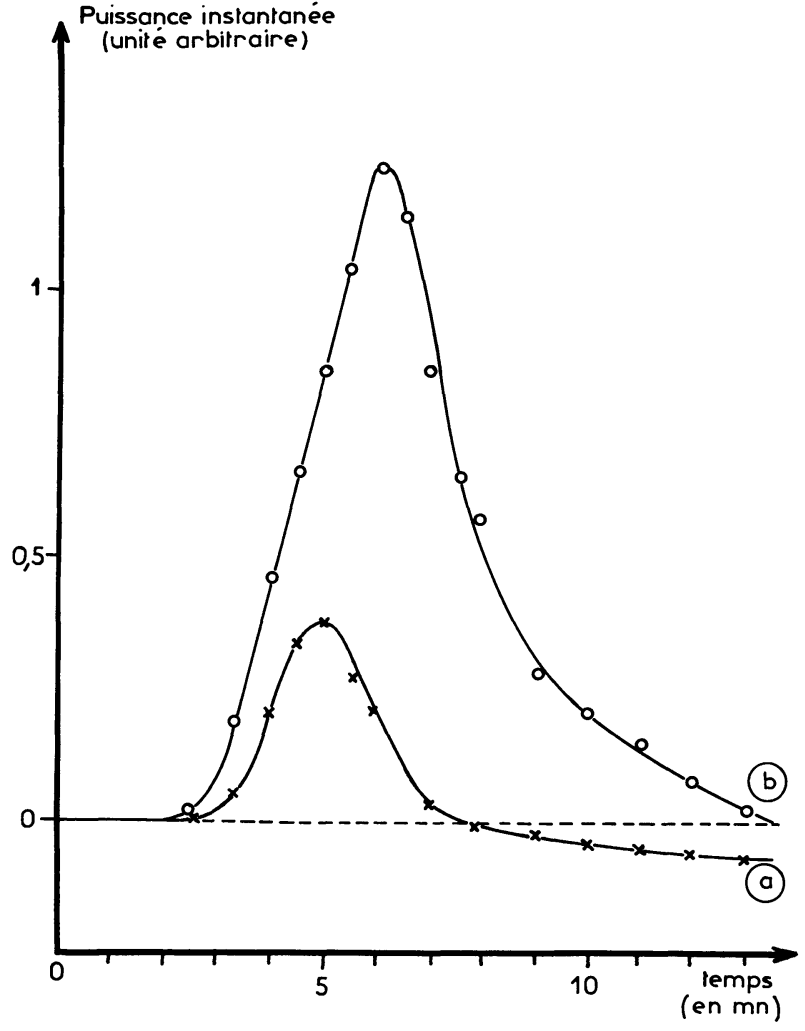

Fig. 4. - La pente $\frac{\mathrm{d} \Delta T}{\mathrm{~d} t}$, relative aux courbes de la figure 3, est proportionnelle à la puissance instantanée récupérée par l'huile lors de la cristallisation du sel; ces courbe démontrent l'intérêt de l'échangeur dynamique à contact direct.

The slope $\frac{\mathrm{d} \Delta T}{\mathrm{~d} t}$ is proportional to the instantaneous power given to the oil at the phase transition of molten salt. This curves show the interest of a dynamic heat exchange with direct contact.]

faciale [22] avec accroissement de pression dans les creux les approfondissant et diminution de pression sur les crêtes les amplifiant. Les sommets des crêtes présentent alors un très faible rayon de courbure $R$; le rapport $k$ (= surface d'échange/volume de matière) qui varie comme $1 / R$ devient très grand à ce niveau. Ainsi, les sommets des crêtes apparaissent comme les lieux privilégiés de la cristallisation; il se produirait alors une séparation des phases solides et liquides avec écrêtage des sommets. Les cristallites sont entraînés par le vortex existant dans l'huile avec création d'une zone diphasique sel solide-huile. Le processus se poursuit jusqu'à ce que l'interface sel liquide-mélange diphasique se solidifie du fait de la cinétique de refroidissement imposée par la programmation du four.

La courbe de refroidissement de l'ensemble (Fig. 2) met très nettement en évidence ce processus dû à l'apparition de la zone diphasique. Les courbes des figures 3 et 4 mettent en évidence l'importance du mécanisme. On remarque que l'accroissement $\Delta T$ est maximum dans le cas de l'échange dynamique avec 


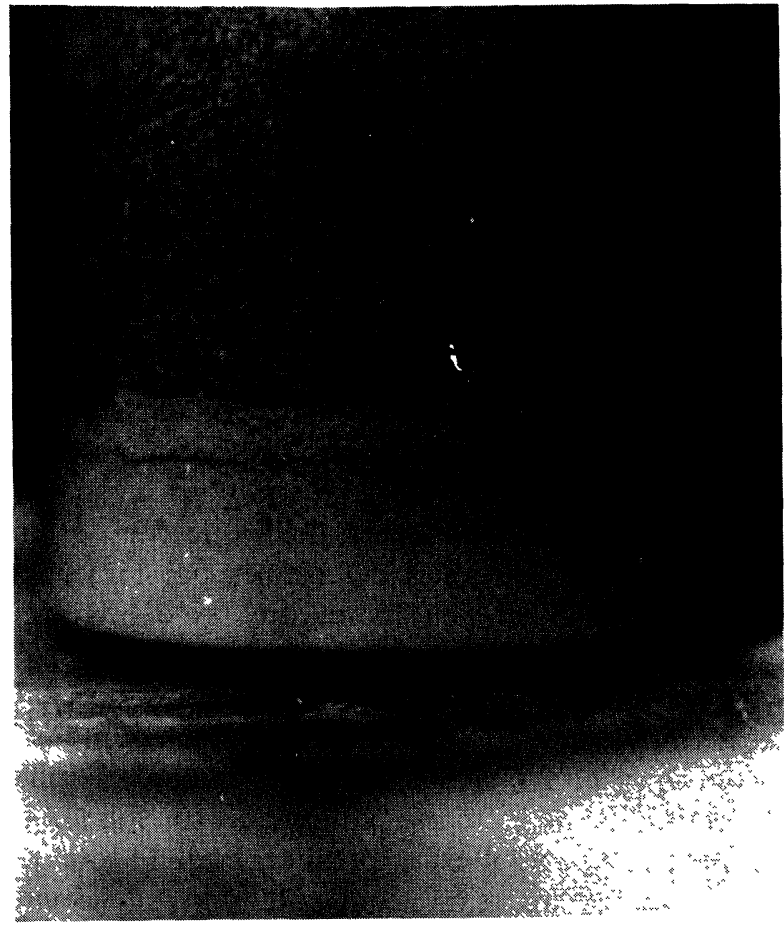

Fig. 5. - Photographie de l'enceinte 1 après le refroidissement avec l'agitateur situé au niveau de l'interface. On note une granulométrie relativement homogène pour les cristallites entraînés dans le vortex (voir Fig. 6). On remarque, dans le fond de l'enceinte, le sel qui s'est solidifié de façon homogène à l'abri de la perturbation créée par l'agitateur; au-dessus se trouve une zone poreuse due à des coalescences importantes entre particules avec, à la partie supérieure, une zone de dépôt des cristallites.

[Photography of first container after freezing for stirrer situated at the interface oil-molten salt. We observe that salt particle size is not very dispersed. We see, at the low part of the container, an homogeneous solid salt, above there is a porous zone produced by coalescence of molten salt drop. Above the porous zone, we see a sediment part and mixture of oil-solid salt particles.]

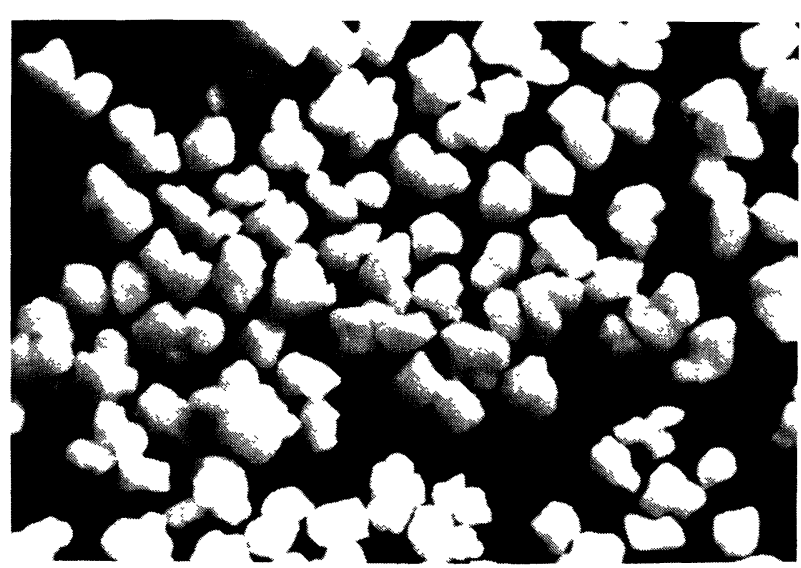

Fig. 6. - Macrophotographie des particules obtenues par agitation au niveau de l'interface huile-sel fondu. On note une granulométrie relativement homogène, le diamètre moyen étant de l'ordre de $1 \mathrm{~mm}$.

[Photography of particles obtained when the stirrer is situated at the interface oil-molten salt. The diameter is around $1 \mathrm{~mm}$.] cristallite; la puissance instantanée est environ le triple de celle récupérée dans la configuration 2 alors que seule une partie du sel a donné lieu à formation de cristallites. La photographie de la figure 5 montre le mélange diphasique en mouvement dans le vortex créé par l'agitateur. On remarque, dans le fond de l'enceinte, la partie solidifiée de sel qui n'a pas donné lieu à formation de cristallites; la photographie de la figure 6 montre que les cristallites possèdent une granulométrie relativement homogène.

Cette première série d'expériences démontre la faisabilité d'un échangeur dynamique à contact direct ; aussi, nous pouvons envisager la possibilité de mettre en œuvre un processus d'injection du sel liquide dans le fluide caloporteur.

3. Etude de l'injection du sel fondu dans le fluide caloporteur : échangeur dynamique à contact direct. 3. 1 DESCRIPTION DE L'ENSEMBLE EXPÉRIMENTAL. - Le schéma de la figure 7 et la photographie de la figure 8 donnent le montage de l'ensemble expérimental. On dispose d'une cuve en acier inoxydable de 20 litres pouvant supporter une pression de $10 \mathrm{~kg} / \mathrm{cm}^{2}$ dans laquelle se trouve le sel fondu avec une couverture d'huile puis d'azote. Cet ensemble est placé dans un

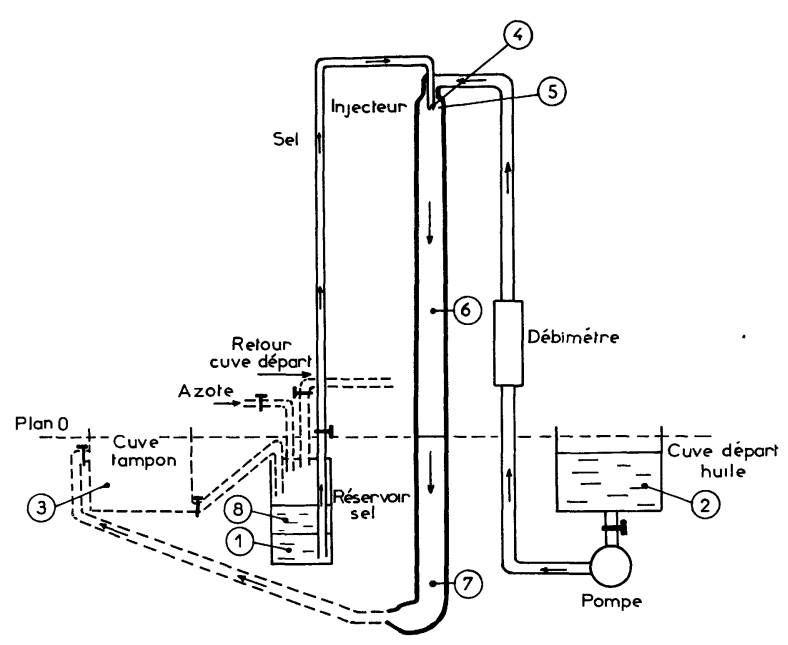

Fig. 7. - Schéma de principe de l'échangeur dynamique à contact direct par injection de sel fondu dans l'huile "Gilotherm-A.L.D. ". Le sel 1 avec sa couverture d'huile 8 est injecté dans la colonne d'échange par la surpression d'azote. Le mélange diphasique solideliquide est récupéré dans la cuve tampon 3 ouverte à l'atmosphère. On dispose de quatre points de mesures de la température : 4. Température du fluide injecté $: T_{\mathrm{si}} ; 5$. Température du fluide caloporteur à l'entrée : $T_{\mathrm{he}} ; 6$. Température du mélange au milieu de la colonne $: T_{\mathrm{hm}} ; 7$. Température du mélange au bas de la colonne : $T_{\text {hs }}$.

[Principle of the dynamic heat exchanger with direct contact by injection of molten salt in an organic oil. The molten salt (1) is injected in the heat exchanger by a nitrogen pressure. The mixture oil-solid salt is received in a container (3) open to the atmosphere. In the heat exchanger we have four points of temperature measure : $4: T_{\mathrm{si}}$, temperature of molten salt at the injection point; $5: T_{\text {he }}$, oil temperature at the up part of the heat exchanger ; $6: T_{\mathrm{hm}}$, oil temperature at the intermediate part $; 7: T_{\mathrm{hs}}:$ oil temperature at the low part.] 


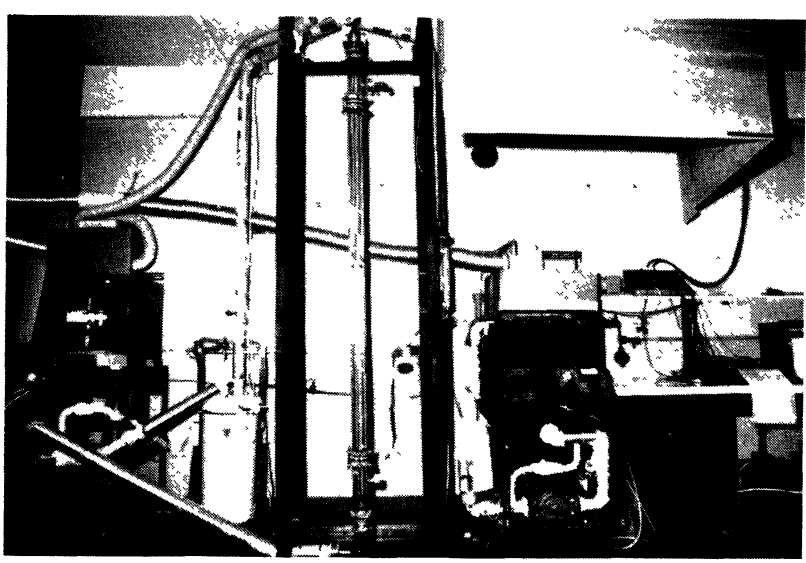

Fig. 8. - Montage expérimental de l'ensemble d'injection.

[Experimental arrangement of the heat exchanger.]

four régulé permettant de conserver les deux liquides à une température préalablement fixée. Une surpression d'azote $P_{i}$ (pression d'injection) permet d'injecter le sel puis l'huile dans la colonne d'échange (hauteur : $280 \mathrm{~cm}$; diamètre $: 5 \mathrm{~cm})$. La canalisation d'injection, en acier inoxydable (diamètre : $0,6 \mathrm{~cm}$ ), est maintenue en température par un enroulement chauffant afin d'éviter toute possibilité de cristallisation durant l'injection.

Le fluide caloporteur est mis en température dans une cuve en acier inoxydable de 100 litres placée dans un four régulé à une température fixée au préalable. Il est mis en mouvement au moyen d'une pompe; le débit est mesuré avec un débitmètre placé sur la colonne ascendante du fluide caloporteur.

Afin de limiter les pertes latérales de chaleur, la colonne d'échange, en pyrex, est placée à l'intérieur d'un manchon également en pyrex. L'air compris entre le manchon et la colonne joue le rôle d'isolant thermique; il circule à contre-courant sous l'effet de la convection naturelle.

Avant de procéder à l'injection du sel, la colonne d'échange est mise en température par le fluide caloporteur circulant en circuit fermé, le fluide en sortie de colonne est renvoyé par un circuit secondaire (non figuré sur le schéma) vers la cuve de départ. On estime que cette opération est terminée lorsque les températures d'entrée et de sortie de l'huile dans la colonne sont égales.

On ferme alors le circuit secondaire, l'huile en sortie de colonne est envoyée dans la cuve tampon puis, quelques secondes après, on ouvre le circuit d'injection de sel en maintenant constante la pression d'injection d'azote mesurée par un manomètre. Le mélange d'abord diphasique liquide-liquide puis diphasique solide-liquide s'écoule dans la colonne puis est évacué vers la cuve tampon. On termine en injectant l'huile de couverture du sel qui permet une vidange complète du tube d'injection en vue de l'injection suivante.

Lorsque l'injection est terminée, on porte le mélange huile-sel solide à une température supérieure au point de fusion du sel $\left(T>142{ }^{\circ} \mathrm{C}\right)$; le bas de la cuve constitué de sel liquide est ensuite renvoyé dans la cuve de sel avec de l'huile; le reste de l'huile est renvoyé vers la cuve de départ en transitant par la cuve de sel. Le processus peut alors recommencer soit à d'autres températures pour le sel ou pour l'huile, soit à d'autres pressions d'injection.

3.2 DéTERMINATION DU DÉBIT DE SEL EN FONCTION DE LA PRESSION D'INJECTION. - Il n'est pas possible de mesurer expérimentalement le débit de sel dans l'injecteur ; en effet, il n'existe pas à notre connaissance de débitmètre à sel fondu. Aussi, nous avons été amenés à élaborer un modèle théorique comportant des paramètres ajustables déterminés par l'injection de fluides dont on peut connaître les débits réels (huile et eau).

Le sel est injecté dans la colonne d'échange à la vitesse moyenne $\left\langle v_{\mathrm{i}}\right\rangle$ à laquelle correspond un débit moyen $\psi_{\mathrm{i}}=\rho_{\mathrm{i}} S_{\mathrm{i}}\left\langle v_{\mathrm{i}}\right\rangle, S_{\mathrm{i}}$ étant la section de l'orifice d'injection et $\rho_{\mathrm{i}}$ la masse volumique du fluide. Le moteur de l'injection est la pression $P_{\mathrm{i}}$ de l'azote que l'on envoie à pression constante dans l'enceinte. Lorsque l'écoulement est établi, le fluide soumet le tube à des forces qui sont de deux types : les forces $\mathbf{F}_{\mathbf{S}}$ qui s'exercent même si le fluide est immobile et les forces $\mathbf{F}_{\mathrm{K}}$ associées au mouvement du fluide. Il est classique [23] d'exprimer le module de ces dernières par le produit d'une surface caractéristique, d'une énergie cinétique caractéristique ramenée à l'unité de volume du fluide et d'un nombre sans dimension : le coefficient de friction $f$.

Dans le cas de l'écoulement dans un tube, on pourra donc écrire :

$F_{\mathbf{K}}=(2 \Pi R L)\left(\frac{1}{2} \rho_{\mathrm{i}}\left\langle v_{\mathrm{i}}\right\rangle^{2}\right) f$

$R$ étant le rayon du tube et $L$ sa longueur.

Si $P_{\mathrm{s}}$ est la pression de sortie et $P_{\mathrm{e}}$ la pression d'entrée dans le tube d'injection, $z_{\mathrm{s}}$ et $z_{\mathrm{e}}$ les ordonnées respectives de l'entrée et de la sortie du tube, les forces qui s'exercent sur le fluide et qui équilibrent $\mathbf{F}_{\mathbf{K}}$ peuvent s'écrire en module :

$F_{\mathrm{K}}^{\prime}=\left[\left(P_{\mathrm{e}}-P_{\mathrm{s}}\right)+\rho_{\mathrm{i}} g\left(z_{\mathrm{e}}-z_{\mathrm{s}}\right)\right] \Pi R^{2}$.

On en déduit alors l'expression du coefficient de friction de Fanning :

$f=\left(\frac{R}{2 L}\right) \times \frac{\left(P_{\mathrm{e}}+\rho_{\mathrm{i}} g z_{\mathrm{e}}\right)-\left(P_{\mathrm{s}}+\rho_{\mathrm{i}} g z_{\mathrm{s}}\right)}{\frac{1}{2} \rho_{\mathrm{i}}\left\langle v_{\mathrm{i}}\right\rangle^{2}}$

qui peut être déterminée expérimentalement.

Lorsque l'écoulement est parfaitement établi, on montre que le coefficient $f$ peut s'exprimer uniquement en fonction du nombre de Reynolds :

$R_{\mathrm{e}}=\frac{2 R\left\langle v_{\mathrm{i}}\right\rangle}{v_{\mathrm{i}}}$

( $v_{\mathrm{i}}$ étant la viscosité cinématique du fluide). 
Les courbes donnant la fonction $f=f\left(R_{\mathrm{e}}\right)$ sont données dans la littérature [24, 25]. Pour des nombres de Reynolds compris entre $2 \times 10^{3}$ et $10^{5}$ correspondant à un écoulement turbulent, le coefficient $\mathrm{f}$ est donné par la relation de Blasius [26]

$f=\frac{0,0791}{R_{\mathrm{e}}^{1 / 4}}$

relation valable pour des tubes ne présentant aucune rugosité.

A partir des équations (4) et (5), on en déduit la vitesse moyenne $\left\langle v_{\mathrm{i}}\right\rangle$ et le débit de fluide injecté $\psi_{\mathrm{i}}$ :

$$
\begin{aligned}
\left\langle v_{\mathrm{i}}\right\rangle & =4,7056\left(\frac{R^{5}}{v_{\mathrm{i}}}\right)^{1 / 7} \times \\
\times & {\left[\frac{\left(P_{\mathrm{e}}+\rho_{\mathrm{i}} g z_{\mathrm{e}}\right)-\left(P_{\mathrm{s}}+\rho_{\mathrm{i}} g z_{\mathrm{s}}\right)}{\rho_{\mathrm{i}} L}\right]^{4 / 7} } \\
\psi_{\mathrm{i}}= & 4,7056 S_{\mathrm{i}}\left(\frac{R^{5} \rho_{\mathrm{i}}^{3}}{v_{\mathrm{i}}}\right) \times \\
\times & {\left[\frac{\left(P_{\mathrm{e}}+\rho_{\mathrm{i}} g z_{\mathrm{e}}\right)-\left(P_{\mathrm{s}}+\rho_{\mathrm{i}} g z_{\mathrm{s}}\right)}{L}\right]^{4 / 7} . }
\end{aligned}
$$

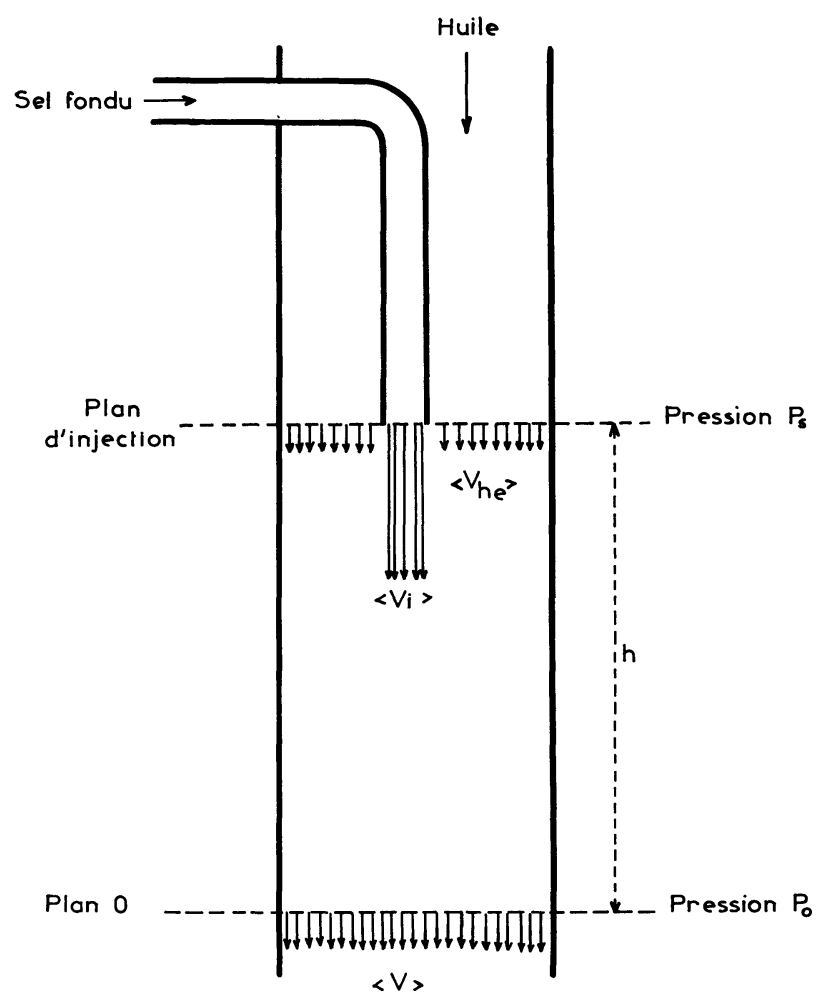

Fig. 9. - Schéma simplifié de l'injecteur tel Bird et al. [23]. Les écoulements sont turbulents et les profils de vitesses supposés plats. A la distance $h$ de l'injecteur, on admet que l'écoulement plats. A la distance $h$ de l'injecteur, on admet que l'écoulement diphasique stationnaire est caractérisé par une vitesse moyenne $\langle v\rangle$.

[The principle of injector as Bird and al. [23]. The flows are turbulent and velocity profiles are flat. At the distance $h$ of the injector, we assume that the two-phase flow is characterized by the velocity $\langle v\rangle$.]
Tels Bird et al. [23], considérons alors un schéma simplifié de l'injecteur liquide-liquide (Fig. 9); on admet qu'au niveau 0 , situé à la distance $h$ de l'injecteur, l'écoulement diphasique est stationnaire et que la pression $P_{0}$ est égale à la pression atmosphérique. La conservation de la masse permet alors d'écrire :

$\rho S_{\mathrm{T}}\langle v\rangle=\rho_{\mathrm{i}} S_{\mathrm{i}}\left\langle v_{\mathrm{i}}\right\rangle+\rho_{\mathrm{h}}\left(S_{\mathrm{T}}-S_{\mathrm{i}}\right)\left\langle v_{\mathrm{he}}\right\rangle$

$\rho$ étant la masse volumique moyenne, $S_{\mathrm{T}}$ la section de la colonne d'échange, $\langle v\rangle$ la vitesse moyenne dans la colonne, $\rho_{\mathrm{h}}$ la masse volumique de l'huile et $\left\langle v_{\mathrm{he}}\right\rangle$ la vitesse moyenne du fluide caloporteur au niveau de l'injecteur. On posera :

$\frac{S_{\mathrm{T}}}{S_{\mathrm{i}}}=k \quad$ et $\quad \frac{\left\langle v_{\mathrm{i}}\right\rangle}{\left\langle v_{\mathrm{he}}\right\rangle}=\frac{\rho_{\mathrm{h}}}{\rho_{\mathrm{i}}} \frac{\psi_{\mathrm{i}}}{\psi_{\mathrm{h}}}(k-1)$

$\psi_{\mathrm{h}}$ étant le débit du fluide caloporteur.

On en déduit la masse volumique moyenne $\rho$ et la vitesse moyenne $\langle v\rangle$.

$$
\begin{aligned}
& \langle v\rangle=\frac{\left\langle v_{\mathrm{i}}\right\rangle}{k}\left(1+\frac{\rho_{\mathrm{i}} \psi_{\mathrm{h}}}{\rho_{\mathrm{h}} \psi_{\mathrm{i}}}\right) \\
& \rho=\rho_{\mathrm{i}}\left(1+\psi_{\mathrm{h}} / \psi_{\mathrm{i}}\right) /\left(1+\rho_{\mathrm{i}} \psi_{\mathrm{h}} / \rho_{\mathrm{h}} \psi_{\mathrm{i}}\right) .
\end{aligned}
$$

En négligeant les forces de frottement sur la colonne d'échange, on peut écrire :

$$
\begin{aligned}
\psi_{\mathrm{i}}\left\langle v_{\mathrm{i}}\right\rangle+\psi_{\mathrm{h}}\left\langle v_{\mathrm{h}}\right\rangle-\psi\langle v\rangle+ \\
+\left(P_{\mathrm{s}}-P_{0}\right) S_{\mathrm{T}}+\rho g h S_{\mathrm{T}}=0 .
\end{aligned}
$$

En tenant compte des relations (10), (11), (12), on en déduit :

$$
\begin{aligned}
P_{\mathrm{s}}= & P_{0}-\rho g h-\left(k \psi_{\mathrm{i}}^{2} / \rho_{\mathrm{i}} S_{\mathrm{T}}^{2}\right) \times \\
& \times\left[1+(1 / k-1)\left(\rho_{\mathrm{i}} / \rho_{\mathrm{h}}\right)\left(\psi_{\mathrm{h}} / \psi_{\mathrm{i}}\right)^{2}\right. \\
& \left.-(1 / k)\left(1+\psi_{\mathrm{h}} / \psi_{\mathrm{i}}\right)\left(1+\rho_{\mathrm{i}} \psi_{\mathrm{h}} / \rho_{\mathrm{h}} \psi_{\mathrm{i}}\right)\right] .
\end{aligned}
$$

Connaissant $P_{\mathrm{e}}$ et $P_{0}$, les relations (7) et (13) permettent, par itérations successives, de déterminer $P_{\mathrm{s}}$, $\psi_{\mathrm{i}},\left\langle v_{\mathrm{i}}\right\rangle, \rho$ et $\langle v\rangle$.

Toutefois, il ne s'agit que d'un modèle; nous avons admis que le mouvement turbulent était caractérisé par un profil de vitesse plat, que la longueur $L$ était considérée sans rugosité (ce qui nous a permis d'appliquer la formule de Blasius) et que le fluide diphasique était homogène. Aussi, nous avons calé ce modèle sur une série d'expérimentations dans lesquelles le fluide injecté et le fluide caloporteur étaient identiques (huile ou eau). En assimilant $P_{\mathrm{e}}$ à la pression $P_{\mathrm{i}}$ de l'azote, nous déterminons, par application du modèle, la longueur équivalente $L_{\mathrm{eq}}$; nous avons ainsi obtenu une série cohérente de valeurs, la moyenne étant : $L_{\text {eq }}=610 \mathrm{~cm}$. Les courbes des figures 10 et 11 donnent alors le débit et la vitesse du fluide injecté (sel fondu ou huile) dans le fluide caloporteur (huile à $T=100{ }^{\circ} \mathrm{C}$, débit : $260 \mathrm{~g} / \mathrm{s}$ ) en fonction de la pression d'injection $P_{\mathrm{i}}$ et pour différentes températures du fluide 


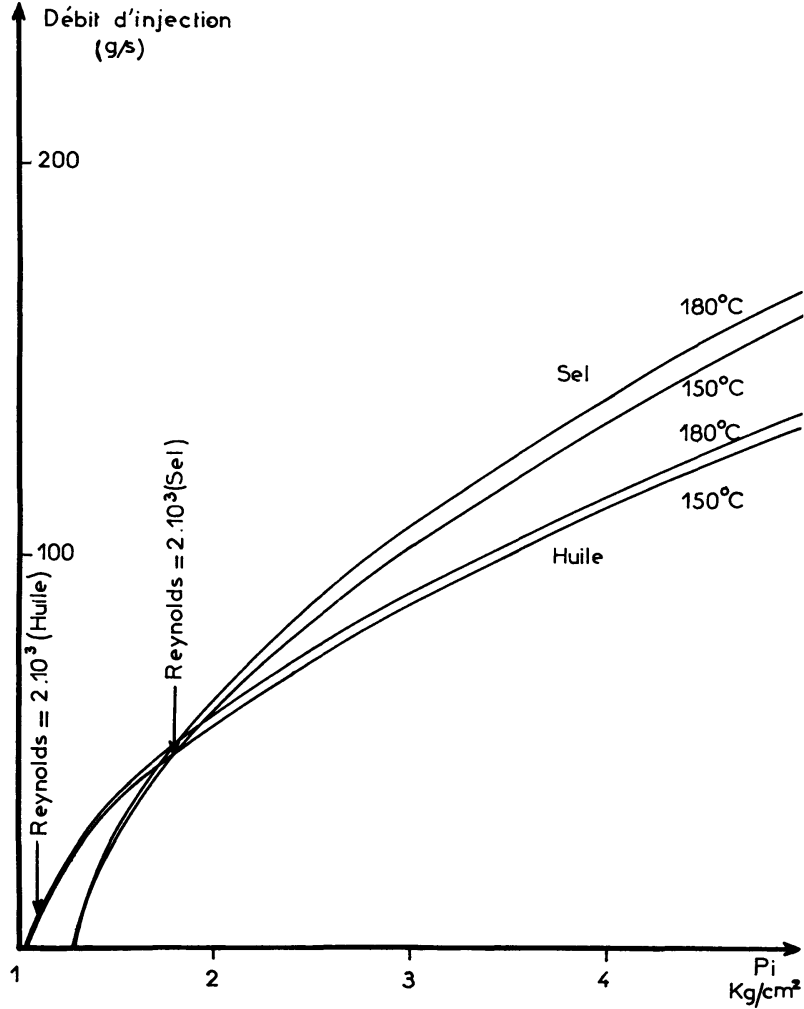

Fig. 10. - A partir du schéma simplifié de l'injecteur, du coeffcient de friction de Fanning et de la formule de Blasius, nous établissons une relation entre la pression d'injection $P_{\mathrm{i}}$ et le débit de fluide injecté après avoir déterminé par étalonnage la longueur $L_{\text {eq }}(\simeq 610 \mathrm{~cm})$ de la canalisation d'injection. On remarque que l'influence de la température sur les caractéristiques des fluides ne modifie que légèrement le débit. La limite inférieure caractérisée par le nombre de Reynolds $\left(R_{\mathrm{e}} \simeq 2 \times 10^{+3}\right)$ correspond à la limite de validité du modèle.

[From the principle of injector, of Fanning coefficient and Blassius formula, we establish a relation between the injection pressure $P_{i}$ and the flow injected for two different fluids (oil and molten salt) and different values of temperature.]

injecté. On remarque que l'influence de la température est faible, elle est principalement due à la variation de la viscosité. Nous avons également noté que le débit d'injection ne dépend que faiblement du débit $\psi_{\mathrm{h}} \mathrm{du}$ fluide caloporteur. Enfin, nous avons indiqué par des flèches les limites inférieures de validité du modèle $\left(R_{\mathrm{e}}>2 \times 10^{3}\right)$.

3.3 DesCription De L'INJeCTeur. - Dans une première série de manipulation, nous avons utilisé un injecteur à orifice de sortie rectangulaire $(1 \mathrm{~mm} \times 12,5 \mathrm{~mm})$. Ce dernier présentait l'inconvénient de s'obstruer en fin d'injection de sel; aussi, avant chaque cycle d'injection, il était nécessaire de préchauffer la colonne d'échange pour éliminer par fusion le résidu de sel solide.

Dans une deuxième série de manipulation, nous avons utilisé un injecteur cylindrique de diamètre :

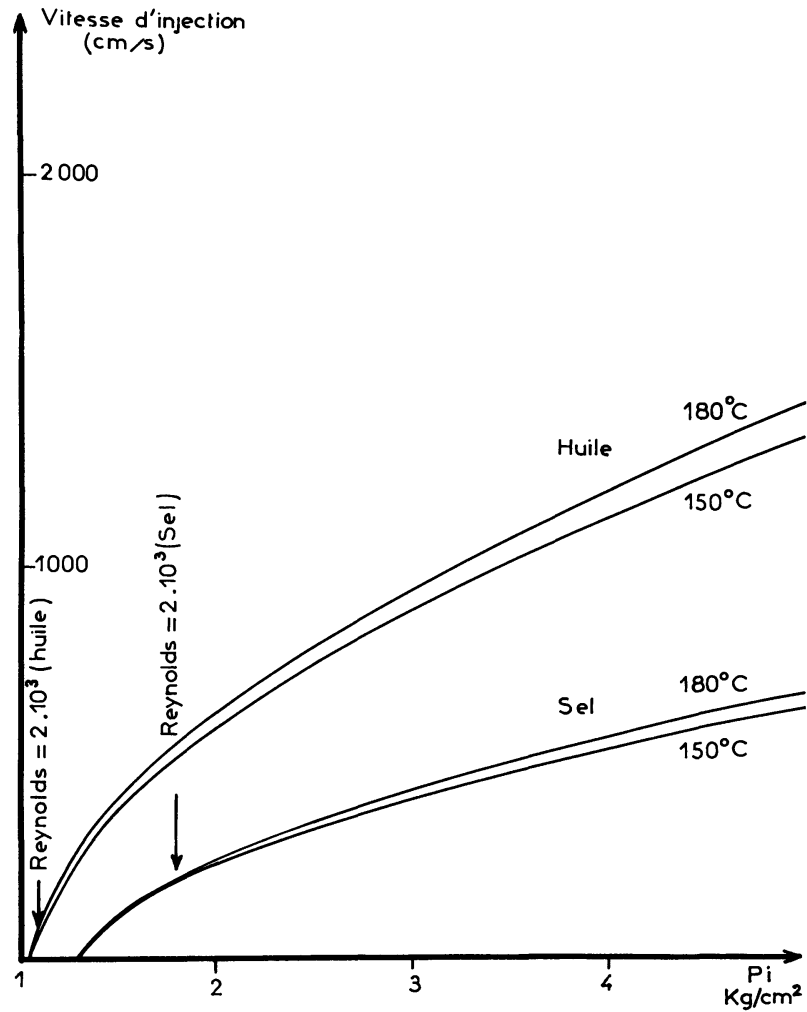

Fig. 11. - Relation entre la vitesse du fluide injecté au niveau de l'injecteur et la pression d'injection $P_{\mathrm{i}}$ déterminée dans les mêmes conditions que celles de la figure 10 .

[Relation between fluid velocity at the injection point and the pressure $P_{\mathrm{i}}$.]

$\varphi=4 \mathrm{~mm}$ avec lequel nous avons obtenu les résultats faisant l'objet du présent travail. La photographie des figures 12 et 13 donne la forme du jet dans le cas de l'huile et du sel. On constate, comme le prévoit la théorie [22], que dans le cas de l'injection de l'huile dans de l'huile, le jet est conique; il est expérimentalement admis que l'angle d'ouverture du cône est de $25^{\circ}$ à $30^{\circ}$ lorsque le fluide recevant l'injection est immobile; en ce qui nous concerne, le fluide caloporteur, en écoulement turbulent à co-courant avec le jet, provoque la fermeture du cône qui présente alors un angle d'ouverture de $20^{\circ}$. On constate également, dans le cas de l'injection du sel dans le fluide caloporteur, qu'il n'y a pas formation d'un cône de turbulence comme précédemment mais plutôt un comportement analogue à celui d'un jet de fuel dans de l'air; on note l'existence d'une instabilité prenant naissance dans le jet, le remontant et le fracturant avec création d'une atomisation. L'interface sel-huile est nettement marqué, la cohésion du jet étant assurée, avant sa fracture, par les tensions interfaciales développées à ce niveau. Les photographies de la figure 14 donnent la forme des cristallites pour des pressions d'injection : $P_{\mathrm{i}}=2,15 \mathrm{~kg} / \mathrm{cm}^{2}$ et $P_{\mathrm{i}}=2,9 \mathrm{~kg} / \mathrm{cm}^{2}$ (pression absolue). 


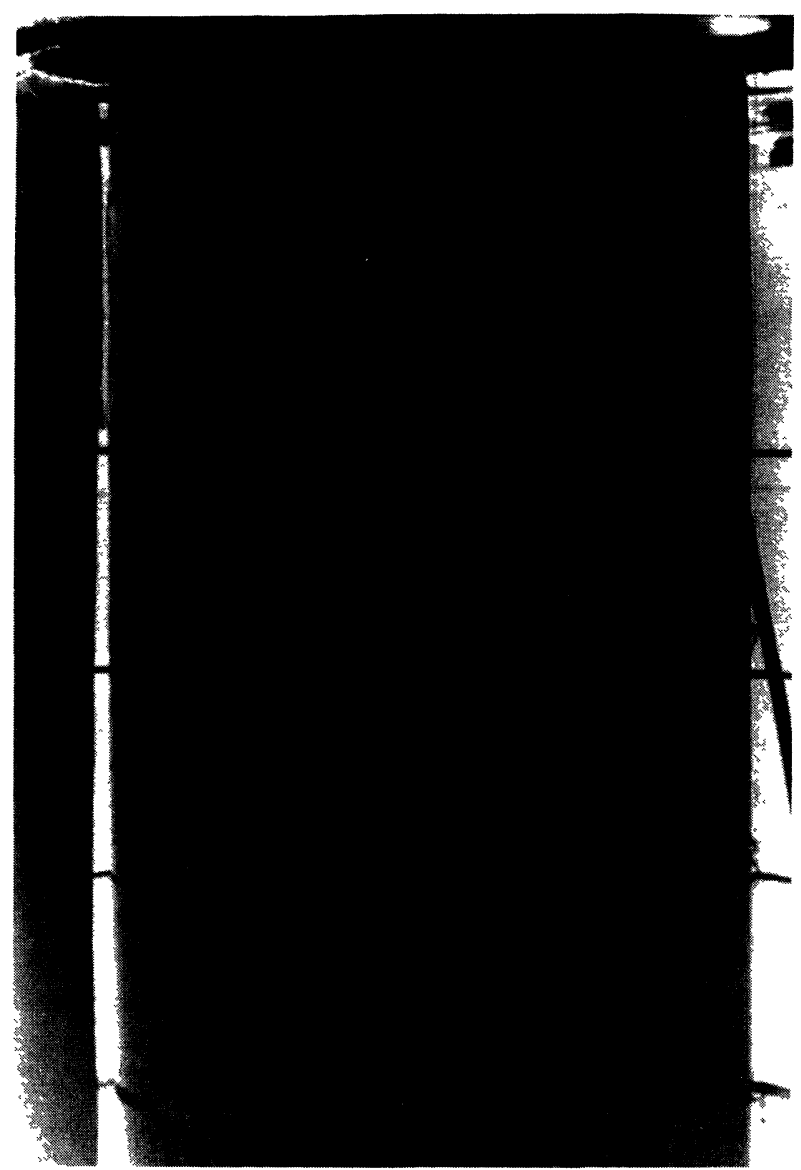

Fig. 12. - Photographie du jet d'huile chaude $\left(T \simeq 150^{\circ} \mathrm{C}\right)$ dans de l'huile froide $\left(T \simeq 100^{\circ} \mathrm{C}\right)$. Les traits horizontaux tracés sur un panneau situé en arrière de la colonne permettent de visualiser la forme du jet. On obtient un cône d'ouverture $\alpha \simeq 20^{\circ}$.

[Photography of an oil jet $\left(T \simeq 150^{\circ}\right)$ in the oil flow $\left(T \simeq 100^{\circ}\right)$. The horizontal dash give a visualization of the jet; we obtain a cone of aperture $\alpha \simeq 20^{\circ}$.]

4. Résultats expérimentaux. - Nous donnons dans le présent travail les résultats obtenus avec deux injections types dont les caractéristiques sont :

Première injection :

Pression d'injection : $P_{\mathrm{i}}=2,150 \mathrm{~kg} / \mathrm{cm}^{2}$.

Température initiale du fluide caloporteur : $T_{\text {he }}=100^{\circ} \mathrm{C}$.

Température du sel à l'injection :

$163^{\circ} \mathrm{C}<T_{\text {si }}<180^{\circ} \mathrm{C}$.

Débit de sel : $\psi_{\mathrm{i}}=69 \mathrm{~g} / \mathrm{s}$.

\section{Deuxième injection :}

Pression d'injection : $P_{\mathrm{i}}=2,900 \mathrm{~kg} / \mathrm{cm}^{2}$.

Température initiale du fluide caloporteur : $T_{\text {he }}=79^{\circ} \mathrm{C}$.

Température du sel à l'injection : $T_{\mathrm{si}} \simeq 158^{\circ} \mathrm{C}$.

Débit de sel $: \psi_{\mathrm{i}}=100 \mathrm{~g} / \mathrm{s}$.

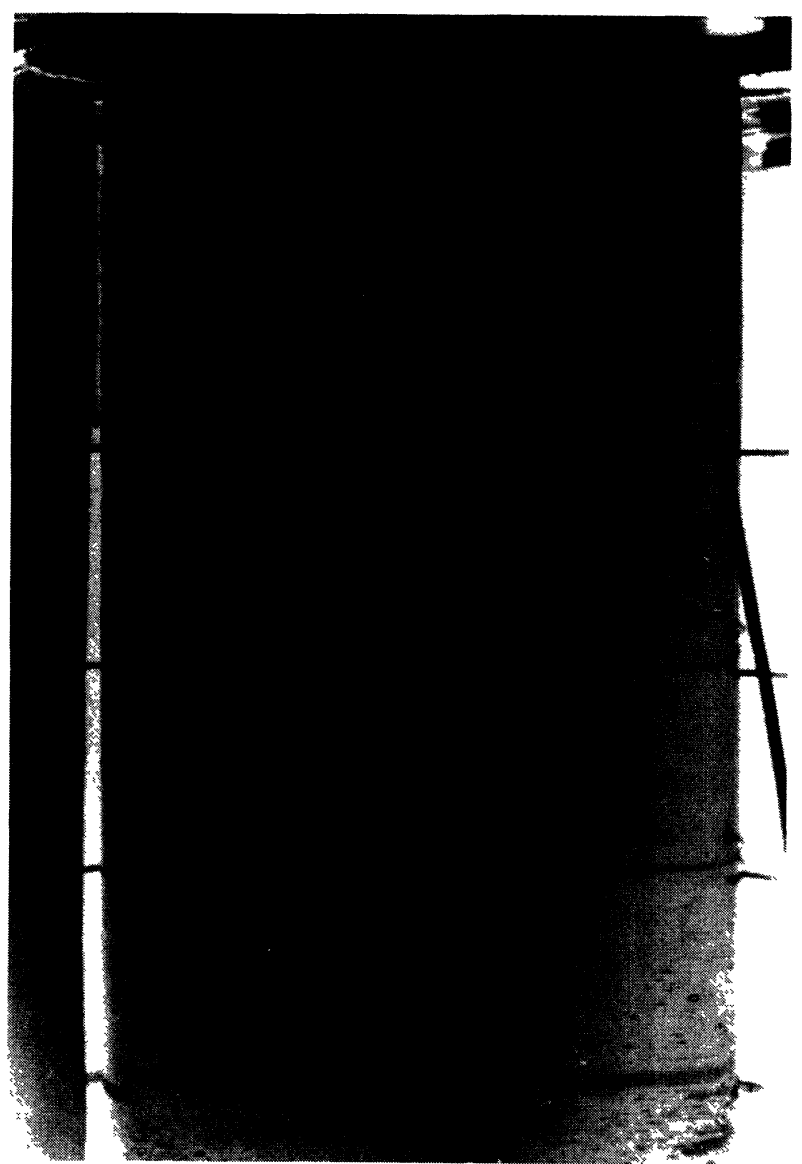

Fig. 13. - Photographie du jet de sel fondu $\left(T \simeq 160^{\circ} \mathrm{C}\right)$ dans l'huile froide $\left(T \simeq 100^{\circ} \mathrm{C}\right)$. On remarque que, contrairement à la figure 12 , le jet n'est pas conique; l'interface est bien marqué du fait de la cohésion du jet assurée par les tensions interfaciales. On note, dans la partie médiane du jet, la présence de l'instabilité ainsi que la fracture du jet dans la zone inférieure. C'est à ce niveau que se situent les principaux phénomènes mis en jeu telles dimensions et formes des particules (voir Fig. 14).

[Photography of a molten salt jet $\left(T \simeq 160^{\circ}\right)$ in an oil flow $\left(T \simeq 100^{\circ}\right)$. Contrarily to figure 12 , the jet is not a cone ; the interface is very well indicated because the cohesion is assured by interfacial strength. We see along the jet the presence of an instability and the breaking of the jet at the low part.]

4.1 ETUde DE la GRANUlOMÉTRIE. - Après chaque injection, nous prélevons dans la cuve-tampon (Fig. 7) un échantillonnage de sel solide. On élimine l'huile par des lavages successifs à l'acétone suivis de séchage à la température ambiante afin d'éviter une modification de la granulométrie par fracture des cristallites. Nous étudions alors la granulométrie de l'injection avec une série de tamis calibrés; les histogrammes obtenus, donnant la concentration en masse en fonction des dimensions, sont donnés par la figure 15.

Nous remarquons très clairement que la première injection correspondant à une faible vitesse d'injection $\left(V_{\mathrm{i}}=280 \mathrm{~cm} / \mathrm{s}\right)$ et à un faible écart entre la température de fusion du sel et la température initiale du 


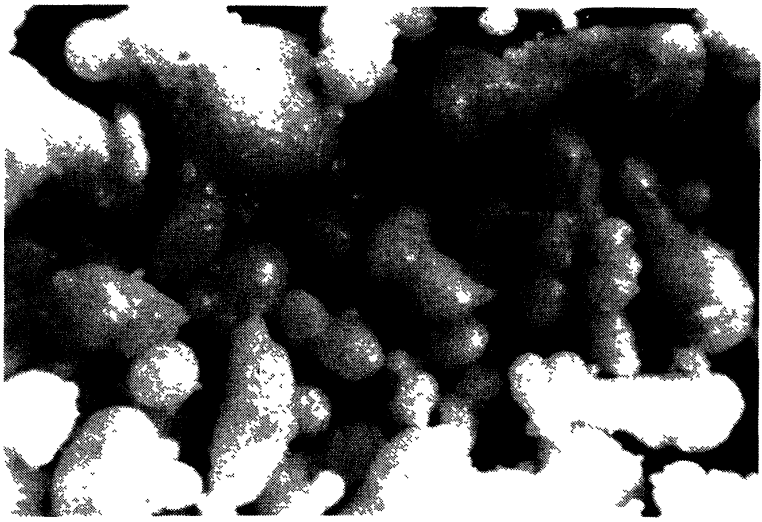

a)

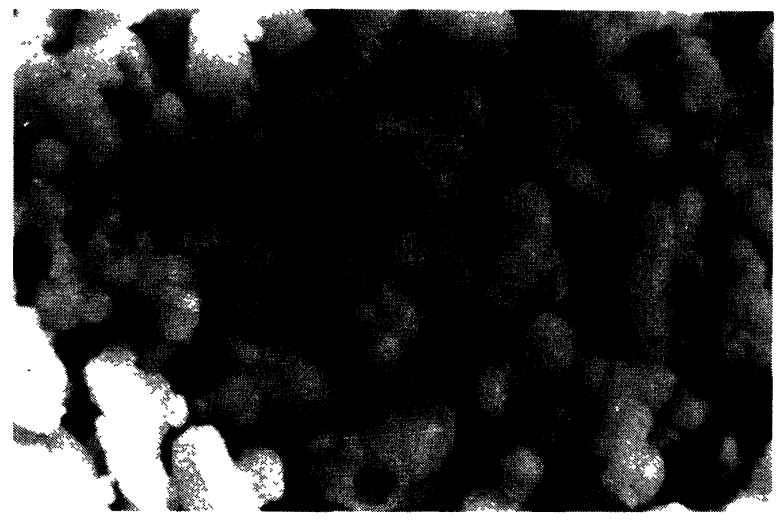

b)

Fig. 14. - Particules recueillies dans la cuve tampon après injection : a) Pression d'injection $P_{\mathrm{i}}=2,150 \mathrm{~kg} / \mathrm{cm}^{2} ; b$ ) Pression d'injection $P_{\mathrm{i}}=2,9 \mathrm{~kg} / \mathrm{cm}^{2}$. On note une granulométrie de base de forme sphérique ou légèrement ovoïde. Cette granulométrie peut donner lieu à des coalescences (voir Fig. 15) plus importantes dans le cas (a) que dans le cas (b).

[Particles obtained after injection for two pressures $P=2.150 \mathrm{~kg} / \mathrm{cm}^{2}$ and $P_{\mathrm{i}}=2.9 \mathrm{~kg} / \mathrm{cm}^{2}$.]
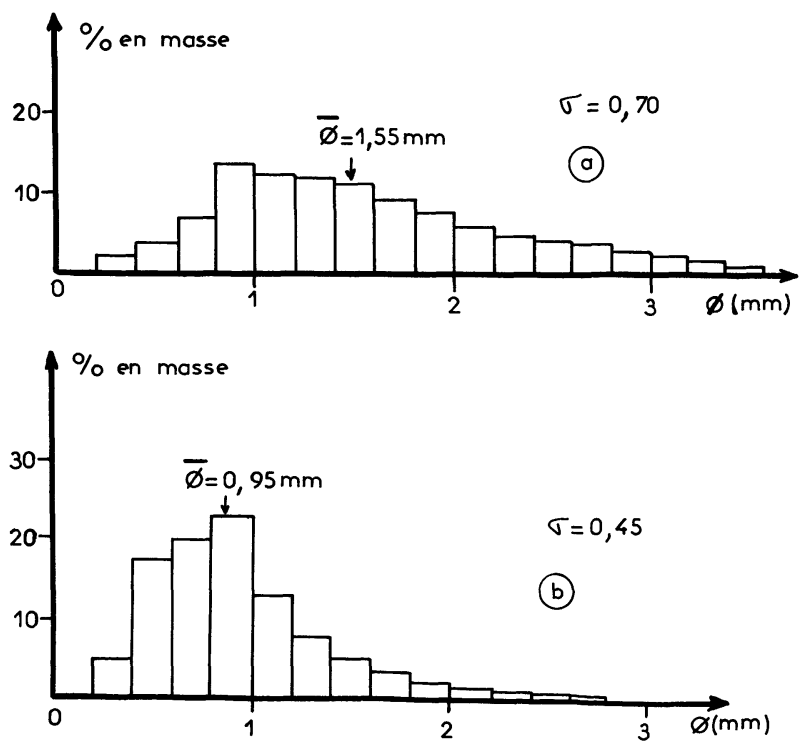

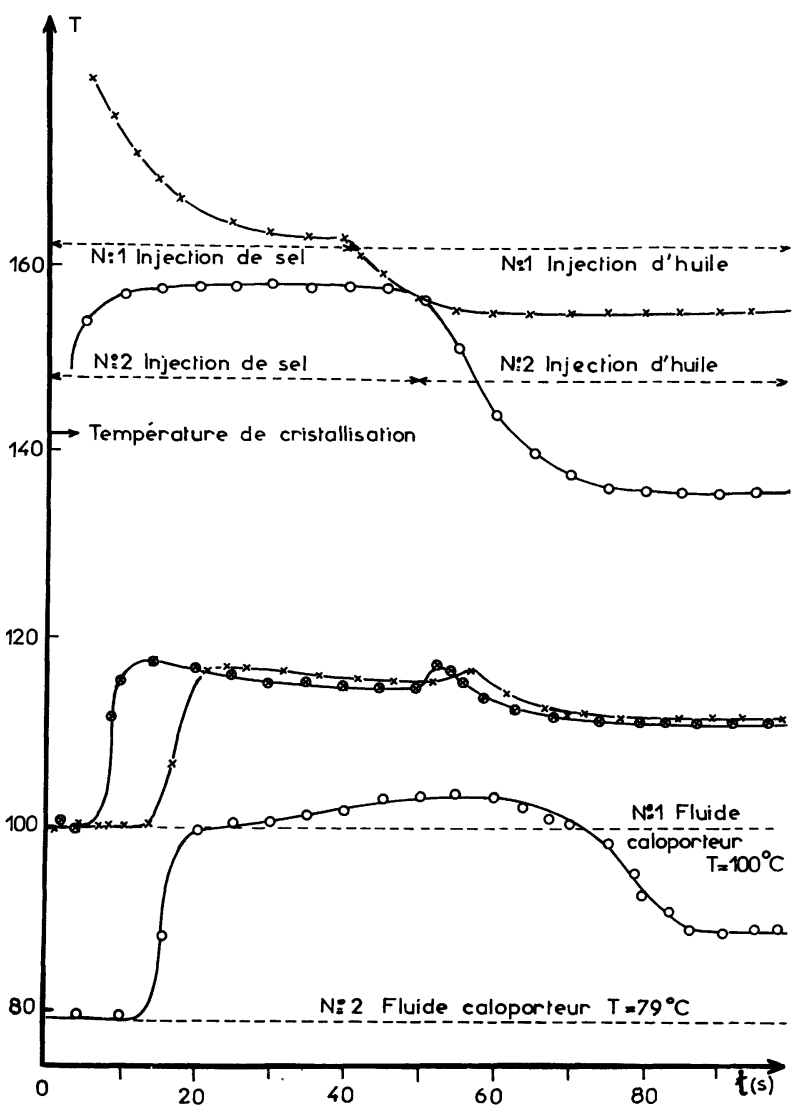

Fig. 16. - Courbe donnant pour les deux injections la température du fluide injecté (sel fondu puis huile), la température d'entrée $T_{\text {he }}$ du fluide caloporteur et la température de sortie du mélange $T_{\mathrm{hs}}$. Pour l'injection $\mathrm{n}^{\circ} 1$, nous donnons également la température du mélange $T_{\mathrm{hm}}$ en milieu de colonne qui est légèrement inférieure à $T_{\mathrm{hs}}$, montrant ainsi que dans ce cas, la puissance n'a pas encore été totalement récupérée après $140 \mathrm{~cm}$ de chute.

[Curves giving, for two injections, the temperature of injected fluid (molten salt and oil) and the temperature of heat transfer fluid (oil) for two points $\left(T_{\mathrm{he}}\right.$ and $\left.T_{\mathrm{hs}}\right)$.]

Fig. 15. - Histogramme donnant pour chaque injection la répartition en masse en fonction des dimensions : $a$ ) Injection à

$P_{\mathrm{i}}=2,150 \mathrm{~kg} / \mathrm{cm}^{2}$;

b) Injection à $P_{\mathrm{i}}=2,9 \mathrm{~kg} / \mathrm{cm}^{2}$. On remarque que l'injection (a) correspond à des particules dont la dimension moyenne est supérieure à celle de l'injection (b). L'importance en concentration des particules de grandes dimensions dans l'injection (a) témoigne des phénomènes de coalescence.

[Mass distribution as a function of size. a) $P_{\mathrm{i}} \simeq 2.15 \mathrm{~kg} / \mathrm{cm}^{2}$, b) $P_{\mathrm{i}}=2.9 \mathrm{~kg} / \mathrm{cm}^{2}$. We see, in case (a), the coalescence phenomena.] 
fluide caloporteur $\left[\left(T_{\mathrm{F}}-T_{\mathrm{he}}\right)=42^{\circ} \mathrm{C}\right]$ donne un histogramme relativement plat (écart-type $\sigma \simeq 0,70$ ) et présentant une forte asymétrie. La deuxième injection, par contre, correspondant à des vitesses d'injection plus grandes $\left(V_{\mathrm{i}} \simeq 400 \mathrm{~cm} / \mathrm{s}\right)$ et des écarts de température plus importants

$\left(T_{\mathrm{F}}-T_{\mathrm{he}}=63^{\circ} \mathrm{C}\right)$

donne un histogramme avec un maximum bien marqué (écart-type $\sigma=0,45$ ) et une asymétrie moins importante.

L'histogramme relatif à la première injection caractérise la présence de particules de grandes dimensions résultant de la coalescence entre particules plus petites ; ce phénomène de coalescence est dû à la mauvaise qualité des transferts thermiques à l'interface sel-huile (faible vitesse et faible écart de température).

4.2 ETUDE DU RENDEMENT DE L'ÉCHANGEUR. - On dispose de quatre points de mesure de la température :

- Température du fluide injecté (sel ou huile) en sortie d'injecteur : $T_{\text {si }}$.

- Température du fluide caloporteur au niveau injecteur : $T_{\text {he }}$.

- Température du mélange au milieu de la colonne : $T_{\mathrm{hm}}$. $T_{\mathrm{hs}}$.

- Température du mélange au bas de la colonne :

Dans ces conditions, nous ne pouvons effectuer que des mesures globales et seule une modification ultérieure de l'ensemble expérimental nous permettra une évaluation plus précise des puissances mises en jeu en fonction de la hauteur d'échange.

Les caractéristiques du mélange eutectique sont données par la littérature [27]; nous retiendrons :

Capacité calorifique du solide : $C_{\mathrm{S}}=0,32 \mathrm{cal} / \mathrm{g} . \mathrm{K}$. Capacité calorifique du liquide : $C_{\mathrm{L}}=0,37 \mathrm{cal} / \mathrm{g} . \mathrm{K}$. Chaleur latente de fusion : $\Delta H_{\mathrm{f}}=20 \mathrm{cal} / \mathrm{g}$.

Masse volumique : $\rho_{\mathrm{i}}=2,082-0,7288 \times 10^{-3} T^{\circ} \mathrm{C}$ en $\mathrm{g} / \mathrm{cm}^{3}$.

Viscosité dynamique :

$\mu=67,57 \times 10^{-3} \exp \left(\frac{2247,1}{T}\right)$ en cp .

Température de fusion : $T_{\mathrm{F}}=142^{\circ} \mathrm{C}$.

La puissance fournie par le sel $P_{\mathrm{f}}$, entre la température $T_{\mathrm{si}}$ d'injection et la température $T_{\mathrm{ss}}$ de sortie en bas de la colonne d'échange, est donnée par la relation :

$$
P_{\mathrm{f}}(\mathrm{kW})=4,18 \times 10^{-3} \psi_{\mathrm{i}}\left[0,37 T_{\mathrm{si}}-0,32 T_{\mathrm{ss}}+12,62\right]
$$

et la puissance récupérée par le fluide caloporteur :

$$
P_{\mathrm{r}}(\mathrm{kW})=4,18 \times 10^{-3} \psi_{\mathrm{h}} \int_{T_{\mathrm{he}}}^{T_{\mathrm{hs}}} C \mathrm{~d} T
$$

$C$ étant la capacité calorifique de l'huile GilothermA.L.D. [28] égale à :

$C=0,445+8 \times 10^{-4} \mathrm{~T}^{\circ} \mathrm{C} \mathrm{cal} / \mathrm{g} \cdot \mathrm{K}$,

les débits $\psi_{\mathrm{i}}$ et $\psi_{\mathrm{h}}$ étant exprimés en $\mathrm{g} / \mathrm{s}$.

Les températures $T_{\mathrm{si}}$ du sel à l'injection et $T_{\mathrm{hs}}$ du mélange à la sortie de la colonne varient avec le temps. Aussi, afin de déterminer les puissances $P_{\mathrm{f}}$ et $P_{\mathrm{r}}$, il est nécessaire de se référer au même élément de volume; à l'instant $t$, il est à la température $T_{\mathrm{si}}(t)$ et à l'instant $(t+h / v)$, il est à la température $T_{\mathrm{ss}}(t+h / v)(h$ étant la hauteur de la colonne d'échange). La relation (14) devient alors :

$$
\begin{aligned}
& P_{\mathrm{f}}(\mathrm{kW})=4,18 \times 10^{-3} \psi_{\mathrm{i}} \times \\
& \quad \times\left[0,37 T_{\mathrm{si}}(t)-0,32 T_{\mathrm{ss}}(t+h / v)+12,62\right] .
\end{aligned}
$$

De même, la relation (15) s'écrit :

$P_{\mathrm{r}}(\mathrm{kW})=4,18 \times 10^{-3} \psi_{\mathrm{h}} \int_{T_{\mathrm{he}}(t)}^{T_{\mathrm{hs}}(t+h / v)} C \mathrm{~d} T$.

Cette hypothèse suppose que durant le temps $t=h / v$, la chaleur ne diffuse pas de façon sensible dans le fluide, c'est-à-dire qu'il y a une statification en température ne se déformant pas avec le temps.

Afin de calculer la puissance $P_{\mathrm{f}}$, il est également nécessaire de connaître la température $T_{\mathrm{ss}}$ du sel solide à la sortie de la colonne d'échange. Nous l'avons assimilée à la température $T_{\mathrm{hs}}$ du mélange diphasique. Le sel est sans doute à une température supérieure à celle du mélange. Toutefois, la puissance $P_{\mathrm{f}}$ ainsi déterminée est supérieure à la puissance réelle et le rendement $K=P_{\mathrm{r}} / P_{\mathrm{f}}$ est alors sous-estimé par rapport au rendement réel.

La figure 16 donne les courbes $T_{\mathrm{si}}$ et $T_{\mathrm{hs}}$ dans le cas des deux injections, le fluide caloporteur étant en haut de colonne à la température initiale $T_{\mathrm{hs}}$ du bas de la colonne. Les tableaux I et II donnent les résultats obtenus.

Pour l'établissement de ces tableaux, nous n'avons pas pris en compte l'énergie dissipée dans la colonne, ce qui explique que dans le deuxième cas, la puissance récupérée $P_{\mathrm{r}}$ croisse avec le temps; cela correspond à la mise en température de la colonne de pyrex.

Tableau I. -

$P_{\mathrm{i}}=2,15 \mathrm{~kg} / \mathrm{cm}^{2}-\Delta T=T_{\mathrm{F}}-T_{\mathrm{he}}=42^{\circ} \mathrm{C}$.

10
15

15
20

20
25

30
35

$\begin{array}{lc}\begin{array}{cc}T_{\mathrm{si}}(t) \\ { }^{\circ} \mathrm{C}\end{array} & T_{\mathrm{hs}}\left(t+\frac{h}{v}\right) \\ - & { }^{\circ} \mathrm{C} \\ 174 & - \\ 169 & 116,7 \\ 166 & 116,3 \\ 164,5 & 115,8 \\ 163,5 & 115,5 \\ 163 & 115,3 \\ 163 & 115,2 \\ & 115\end{array}$

$\begin{array}{ccc}P_{\mathrm{f}}(\mathrm{kW}) & \boldsymbol{P}_{\mathrm{r}}(\mathrm{kW}) & \mathrm{K}=\boldsymbol{P}_{\mathrm{r}} / P_{\mathrm{f}} \\ - & - & - \\ 11,47 & 9,76 & 0,85 \\ 10,98 & 9,53 & 0,87 \\ 10,70 & 9,24 & 0,86 \\ 10,57 & 9,06 & 0,86 \\ 10,48 & 8,95 & 0,85 \\ 10,44 & 8,89 & 0,85 \\ 10,46 & 8,77 & 0,84\end{array}$


Tableau II. -

$P_{\mathrm{i}}=2,9 \mathrm{~kg} / \mathrm{cm}^{2}-\Delta T=T_{\mathrm{F}}-T_{\mathrm{he}}=63^{\circ} \mathrm{C}$.

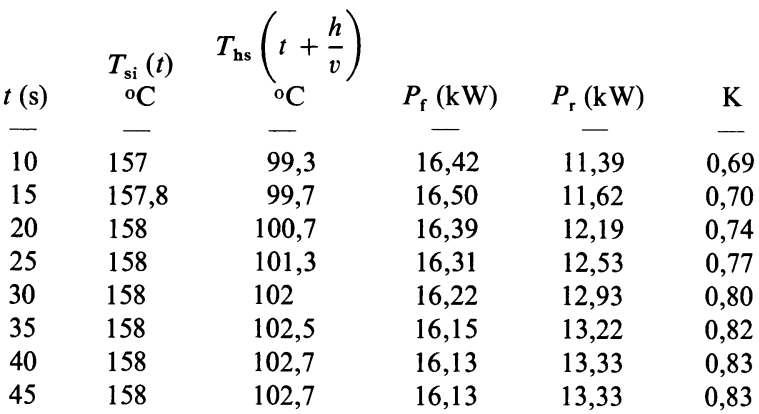

On constate que dans les deux cas, on tend vers un rendement de l'ordre de $85 \%$; il faut toutefois signaler que dans le premier cas, la puissance récupérée en milieu de colonne (ne figure pas sur le tableau) est inférieure à celle récupérée en bas de colonne comme le montre la figure 16. Cela signifie qu'après un échange sur $140 \mathrm{~cm}$, la puissance développée n'a pas totalement été mise en œuvre; par contre, dans le deuxième cas, on obtient la même valeur en milieu et en bas, ce qui signifie que la puissance a été récupérée après $140 \mathrm{~cm}$ d'échange. Cet état est sans doute dû à la granulométrie plus fine de la deuxième injection.

5. Conclusion. - Ce travail nous a permis de montrer que l'échange dynamique à contact direct permet une récupération optimale de la chaleur latente de fusion d'un sel minéral. La puissance thermique est modulable à partir du débit de sel injecté ; cette modulation étant très souple, il suffit de faire varier la pression d'injection $P_{\mathrm{i}}$ du gaz de couverture. Nous poursuivrons ce travail par une étude systématique des conditions d'injection $\left(P_{\mathrm{i}}\right.$ et $\left.\Delta T=T_{\mathrm{f}}-T_{\mathrm{he}}\right)$ afin de déterminer les conditions optimales de fonctionnement de l'échangeur.

Nous avons développé ce travail dans le cadre de l'A.T.P. habitat solaire (C.N.R.S.-P.I.R.D.E.S.) et d'une collaboration avec le Service des Etudes et Recherches de l'Electricité de France. Nous remercions la Société Rhône-Poulenc pour la fourniture du fluide caloporteur.
[1] Ensemble de stockage de la Centrale Solaire d'Odeillo et Projet Them.

[2] Stockage par Sels Fondus - Centrale Themis (CNRS-EDF)

[3] SCHRÖDER, J., Thermal energy storage using fluorides of alkali and alkaline earth metals (148th Meeting of the electrochemical Society. Dallas, Texas) 1975.

[4] Shamsundar, N. and Sparrow, E. M., Storage of thermal energy by solid-liquid phase change temperature drop and heat flux. J. Heat Trans. (1974) 541 .

[5] Muelhbauer, J. C. and Sundelland, J. E., Heat conduction with freezing or melting. Appl. Mech. Rev. 18 (1965) 951.

[6] Chiesa, F. M. and Guthrie, R. I. L., Natural Convective heat transfer, rate during the Solidification and Melting of Alloy and Metal systems. J. Heat Trans. (1974) 377.

[7] Kroeger, P. G. and Ostrach, S., The solution of a two dimensional freezing problem including convection effect in liquid region. Int. J. Heat Mass. Transfer 17 (1974) 1191.

[8] Pantaloni, J. et Velarde, M. G., Algunas cuestiones relativas al almacenamiento termico de la energia mediante sales fundidas. Quim Ind. 22 (1976) 778.

[9] Favre, O. et Pantaloni, J., Etude expérimentale de la stabilité d'un mélange de nitrate et de nitrite soumis à un grand nombre de cycles autour du point de fusion. (Rapport pour le CNRS-EDF dans le cadre du projet T.H.E.M., janvier 1977.)

[10] Desaulty, M., Boyer, J., Huetz, J. et Petit, J. P., Décharge thermique d'un stockage par chaleur latente avec échangeur. Journée d'étude sur le stockage thermique. Société Française des Thermiciens, Paris, avril 1978.

[11] Pantaloni, J., Velarde, M. G., Bailleux, R. et Guyon, E., Sur la convection cellulaire dans les sels fondus près de leur point de solidification. C. R. Hebd. Séan. Acad. Sci. 285 B (1977) 275.

[12] Bardon, J. P., Fusion et solidification périodique dans une plaque verticale : aspects expérimentaux. Journée d'étude sur le stockage thermique. Société Française des Thermiciens, Paris, avril 1978

[13] BRICARD, A., Etude expérimentale de la décharge d'un stockage sur soude fondue. Rapport D.G.R.S.T., mars 1978.

[14] MAYE, J. P., Modèle dynamique de stockage thermique par chaleur latente. Journée d'étude sur le stockage thermique. Société Française des Thermiciens, Paris, avril 1978.

[15] Allard, Etude technico-économique d'un stockage thermique sur matériau fusible encapsulé appliqué au report d'énergie d'heures creuses. Journée d'étude sur le stockage thermique. Société Française des Thermiciens, Paris, avril 1978 .

[16] Marchisio, J., Le Quere, J., Pantaloni, J. et Favre, O., Etude de la cristallisation en écoulement du chlorure de sodium dans du sodium (Rapport contrat EDF, 1978).

[17] BRiQuart, Solidification de l'eau dans un jet de kérozène. Communication table ronde sur le stockage : EDFCNRS, avril 1977, Paris.

[18] GuENOCHE, H. et HUETZ, J., Stockage d'énergie à haut niveau thermique par chaleur latente de fusion. Différents aspects. Journée d'étude sur le stockage thermique. Société Française des Thermiciens, Paris, avril 1978.

[19] DE Montcuir, Etude par analyse thermique différentielle du couple $\mathrm{NaOH}$-Gilotherm. (Communication privée de la Société Rhône-Poulenc).

[20] Dou, H. J. M., Etude de la stabilité thermique du Gilotherm en présence de sel eutectique $\left(\mathrm{NaNO}_{2}-\mathrm{NaNo}_{3}-\mathrm{KNo}_{3}\right)$ par spectrométrie infrarouge. Communication privée, Laboratoire de Chimie organique R-U.D.E.S.A.M. (1978).

[21] Pantaloni, J., Favre, O. et Finiels, G., Etude de la cristallisation d'un sel minéral dans un fluide caloporteur. (Rapport présenté dans le cadre du colloque A.T.P. « Habitat Solaire » P.I.R.D.E.S., Marseille, février 1978.)

[22] LANDAU, L. et Lifchitz, E., Mécanique des fluides (Edition MIR) 1971, p. 166.

[23] Bird, R. B., SteWARD, W. E. and Lightfoot, E. N., Transfer Phenomena (John Wiley and Sons, Inc. New York) 1960.

[24] Moody, L. F., Trans. A.S.M.E. 66 (1944) 671.

[25] McGrabe, W. L. and SMith, J. C., Unit operations of chemical engineering (McGraw-Hill, New York) 1954.

[26] Schlichting, H., Boundary Layer theory (McGraw-Hill, New York) 1955.

[27] Dupont-Hitec, Heat transfer Salt. Explosives department. Wilmington, Delaware 19898.

[28] Notice technique, Gilotherm, A.L.D., Rhône-Poulenc. Chimic Fine. 